\title{
The Cost CHAnNel RECONSIDERED: A COMMENT USING AN IDENTIFICATION-ROBUST APPROACH*
}

\author{
VAsco J. Gabriel \\ Department of Economics, University of Surrey, UK and NIPE-UM (v.gabriel@surrey.ac.uk) \\ Luis F. MARTins \\ Department of Quantitative Methods and UNIDE, ISCTE, Portugal (luis.martins@iscte.pt)
}

This version: January 2010

\begin{abstract}
We re-examine the empirical relevance of the cost channel of monetary policy (e.g. Ravenna and Walsh, 2006), employing recently developed moment-conditions inference methods, including identification-robust procedures. Using US data, our results suggest that the cost channel effect is poorly identified and we are thus unable to corroborate the previous results in the literature.

Keywords: Cost channel; Phillips curve; GMM; Generalized Empirical Likelihood; Weak Identification.

JEL Classification: C22; E31; E32
\end{abstract}

\section{Introduction}

Recent papers argue that fluctuations in short-run nominal interest rates may induce a supplyside dimension to the traditional demand-side transmission mechanism of monetary policy. Indeed, to the extent that firms may need to borrow to finance working capital, changes in interest

\footnotetext{
${ }^{*}$ The authors thank the editor and a referee for insightful comments and suggestions. The first author acknowledges financial support from ESRC grant RES-061-25-0115. The second author acknowledges financial support under grant PTDC/ECO/68367/2006 from the Fundação para a Ciência e Tecnologia. We thank Ron Smith, Paul Levine and participants at the North American Econometric Society Summer Conference 2008 in Pittsburgh and the Econometric Society European Meeting 2008 in Milan for useful comments. We are also indebted to Patrik Guggenberger for discussions on the implementation of GEL procedures. The usual disclaimer applies.
} 
rates potentially impact on firms' marginal cost, thus affecting their pricing decisions. The New Keynesian Phillips Curve (NKPC) then becomes

$$
\pi_{t}=\beta \pi_{t+1}+\lambda\left(\alpha_{c} r_{t}+s_{t}\right)+\varsigma_{t}
$$

with marginal costs $m c_{t}=\alpha_{c} r_{t}+s_{t}$, where $\pi_{t}$ denotes the inflation rate, $r_{t}$ is the nominal interest rate, $s_{t}$ real unit labour costs, $\varsigma_{t}$ is a disturbance term capturing expectational or measurement errors, with $\alpha_{c}$ measuring the magnitude of the cost channel. This effect can cause inflation and nominal interest rates to move in the same direction (rather than the opposite) after a monetary tightening, giving rise to a 'price puzzle' and may thus have important consequences for the conduct of monetary policy, as explored in Ravenna and Walsh (2006, RW henceforth) and Tillmann (2009), for example.

Empirical evidence on the existence of a cost channel comes, to a large extent, from VAR analysis of monetary policy shocks, which indicates the existence of a persistent, 'hump-shaped' estimated response of inflation. Christiano, Eichenbaum and Evans (2005) incorporate this effect in their estimated DSGE model of the US economy, without which estimates of price durations become empirically implausible. On the other hand, Barth and Ramey (2001) provide evidence of such a channel using industry-level US data, while RW and Chowdhury, Hoffmann and Schabert (2006, CHS hereafter) estimate New Keynesian Phillips curves (NKPC) augmented with a cost channel term, obtaining significant interest rate elasticities. More recently, Tillmann (2008), also using a VAR approach based on the implied present-value representation of the NKPC, confirms the importance of the cost channel.

However, this VAR-based stylized fact has been recently challenged by Mojon (2008), who claims that, once shifts in the level of inflation are accounted for, the 'price puzzle' disappears. Also, this author finds that the 'hump-shaped' effect is very sensitive to the sample period considered. Furthermore, Rabanal (2007) finds the cost channel effect to be quantitatively very small in the context of New Keynesian DGSE models estimated with Bayesian methods.

It seems that the empirical relevance of the cost channel is still an open question. Thus, in this paper we re-examine the evidence on the cost channel arising from direct estimation of the NKPC, as in RW and CHS, using US data. We employ recently developed moment-conditions inference methods, namely Generalized Empirical Likelihood (GEL) procedures, which include identification-robust methods. We show that once appropriate methods are put to use, the empirical relevance of the cost channel is feeble and, at best, ambiguous.

Several reasons motivate our approach. Firstly, several authors have questioned the validity 
of early NKPC estimation results, arguing that the key parameters of the NKPC are weakly identified (an excellent discussion can be found in Kleibergen and Mavroeidis, 2009a, see also Mavroeidis, 2005). This may be of great importance, since standard inference will be invalid in the case of weak identification, as shown by Stock and Wright (2000). Given that RW and CHS generalize the usual NKPC, but still rely on standard GMM methods that are not robust to identification problems, it is possible that the identification of the cost channel parameter may also be compromised ${ }^{1}$.

Secondly, the standard GMM estimator used in RW and CHS may deviate substantially from its small sample distribution, as discussed in Hansen, Heaton and Yaron (1996), for example. Moreover, the 2-step GMM estimator is not invariant to transformations of the moment conditions, which means that the results depend on the normalization adopted for the estimation. This problem is quite clear from the results reported by RW (Table 1), with estimates of the cost channel parameter ranging from 1.239 to 11.831. On the other hand, the results of tests of statistical significance stemming from GMM hinge on the weighting matrix used in the estimation. Both RW and CHS estimate the variance-covariance matrix based on a Bartlett kernel with a fixed bandwidth, so it is important to assess how the main results are affected by this particular choice.

Unlike previous papers, we propose instead the use of GEL methods, which include the continuous-updating estimator (CUE) proposed by Hansen et al. (1996). Within this framework, it is possible to compute identification-robust parameter confidence sets, based on appropriate procedures recently proposed by Kleibergen (2005) and extended to a GEL framework by Guggenberger and Smith (2008). A further advantage of Kleibergen's (2005) approach is that it is valid under many weak moments ${ }^{2}$, which, as discussed above, is likely to be the case concerning inference on the NKPC. In addition, GEL-type methods possess higher order efficiency and superior small sample properties when compared to a standard, often biased, GMM estimator, as shown by Newey and Smith (2004) (see also Anatolyev, 2005). Furthermore, GEL estimators do not depend on the normalization adopted for the moment conditions. This will allow us to focus on the economic specifications, rather than on their econometric implementation.

\footnotetext{
${ }^{1}$ Note further that a necessary, though not sufficient, condition for identification of $\alpha_{c}$ in (1) is that $\lambda \neq 0$.

${ }^{2}$ See Newey and Windmeijer (2009).
} 


\section{An identification-robust approach}

We now turn to the estimation of the interest-rate-augmented NKPC for the US, using the orthogonality conditions implied by (1). We use a vector $z_{t}$ of instruments orthogonal to $\varsigma_{t}$, which will typically contain past observations of the variables in (1), but may also include other variables in the information set $\Omega_{t}$. Following Christiano et al. (2005) and CHS, a 'hybrid' version of the NKPC with a cost channel that combines forward and backward-looking behavior can also be defined ${ }^{3}$,

$$
\pi_{t}=\gamma_{f} \pi_{t+1}+\gamma_{b} \pi_{t-1}+\lambda\left(\alpha_{c} r_{t}+s_{t}\right)+\varsigma_{t}
$$

In order to estimate (1) or (2), we employ the CUE and the smoothed EL estimator of Kitamura and Stutzer (1997). For a given $p$-dimensional parameter vector $\boldsymbol{\theta}$, estimation is based on $m \geq p$ moment conditions of the form $E\left[g\left(y_{t}, \boldsymbol{\theta}_{0}\right)\right]=0$, such that $y_{t}=\left(x_{t}, z_{t}\right)$ for some set of variables $x_{t}$ and instruments $z_{t}$. Using Newey and Smith (2004) typology, for a concave function $\rho(v)$ and a parameter vector $\lambda \in \Lambda_{T}(\boldsymbol{\theta})$, the (smoothed) GEL estimator solves the following saddle point problem

$$
\hat{\boldsymbol{\theta}}_{S G E L}=\arg \min _{\boldsymbol{\theta} \in \Re^{p}} \sup _{\lambda \in \Lambda_{T}} T^{-1} \sum_{t=1}^{T} \rho\left[\lambda^{\prime} g_{t T}\left(y_{t}, \boldsymbol{\theta}\right)\right],
$$

where $g_{t T}(\boldsymbol{\theta}) \equiv \frac{1}{2 K_{T}+1} \sum_{k=-K_{T}}^{K_{T}} g_{t-k}(\boldsymbol{\theta})$. When $\rho(v)=-(1+v)^{2} / 2, \hat{\boldsymbol{\theta}}_{S G E L}$ coincides with the CUE, whereas if $\rho(v)=\ln (1-v)$ we have the EL estimator ${ }^{4}$. The latter removes important sources of bias associated with the GMM, namely the correlation between the moment function and its derivative, as well as third-order biases. Furthermore, Anatolyev (2005) shows that even when there is no serial correlation, using smoothing and an appropriate HAC weight matrix, as in Andrews (1991) for example, leads to a reduction in estimation biases.

In addition, GEL estimators are invariant to the normalization of the orthogonality conditions. If one replaces $g$ in $g\left(y_{t}, \boldsymbol{\theta}\right)$ by some $\tilde{g}=A g$, where $A$ is non-singular and depends on $\boldsymbol{\theta}$, $A(\boldsymbol{\theta}), E\left[\tilde{g}\left(y_{t}, \boldsymbol{\theta}_{0}\right)\right]=0$ becomes an alternative formulation of the economic model. There is no economic reason why one should prefer one specification over the other. However, and unlike GEL methods, finite-sample results stemming from two-step GMM estimation vary significantly according to the choice of $A$, a good example being the results reported in Gali and Gertler

\footnotetext{
${ }^{3}$ The reduced form coefficients $\gamma_{f}$ and $\gamma_{b}$ are defined as $\beta \theta \phi^{-1}$ and $\omega \phi^{-1}$, respectively, and $\lambda=(1-\omega)(1-$ $\theta)(1-\beta \theta) \phi^{-1}$, with $\phi=\theta+\omega[1-\theta(1-\beta)]$, where $\beta$ is the subjective discount rate, $\omega$ measures the degree of 'backwardness' and $\theta$ measures price stickiness in a Calvo-type price setting framework.

${ }^{4}$ In the empirical analysis, we set $K_{T}=T^{1 / 3}$, since the optimal bandwidth rate for the truncated kernel used in the Kitamura-Stutzer estimator is $O\left(T^{1 / 3}\right)$ (the results are largely insensitive to the choice of this parameter).
} 
(1999) and, more relevant to our case, the results in RW (Table 1).

For comparability, we estimate the cost channel using the same variables as in RW, consisting of the GDP deflator inflation rate, real marginal cost proxied by non-farm business sector real unit labour costs and the interest rate, measured by the 3-month T-bill rate. Instruments include four lags of: $\pi_{t}, s_{t}, r_{t}$, the CRB commodity price index inflation, wage inflation, the term spread and HP-filtered output gap - see RW for details and data sources.

Table 1 presents results using quarterly data for the period 1960:1-2004:4, obtained with the data-dependent bandwidth selection method of Andrews (1991) with the Bartlett kernel, and sample moments in mean deviation form, thus improving the power of the $J$-test of overidentifying restrictions. Notice that, overall, the two estimators produce consistent and comparable results, as expected. Unlike RW and CHS, however, we found no substantial evidence of a cost channel effect, as the estimates of $\alpha_{c}$ are always insignificant. While it is true that $t$-tests or Wald tests of the hypothesis $\alpha_{c}=1$ are not rejected, this is due to the large standard error associated with the estimates of $\alpha_{c}$. In addition, our point estimates of $\alpha_{c}$ are substantially lower than those presented in RW and, therefore, distant from the benchmark value of $\alpha_{c}=1$. The other parameters are estimated much more precisely and their values are consistent with results reported elsewhere. Moreover, these values appear to be more sensible than those reported in RW. If one includes lagged inflation, estimates of the backward-looking component $\gamma$ range between 0.31 and 0.43 . Restricted versions of the NKPC were also estimated, to evaluate the impact on the other structural coefficients. As can be seen, omitting the cost channel has no sizeable impact on the magnitude of $\beta, \theta$ or $\gamma$.

RW also suggest an ad-hoc strategy of using a smaller set of instruments (instrument set B), supposedly to minimize potential weak identification problems. For comparison purposes, we present in the bottom half of Table 1 estimates using this smaller set (restricted to the first two lags of the variables in the instrument set $\mathrm{A}$, with the exception of the inflation rate and the interest rate, with four lags, as in RW). We can observe that the "B" estimates are very similar to those obtained employing the larger instrument set A, albeit statistically insignificant ${ }^{5}$.

However, the above approach does not address the underlying problems with standard GMM: the 2-step estimator is inconsistent and inference is not valid when based on many - potentially

\footnotetext{
${ }^{5}$ We also considered the block bootstrap introduced by Hall and Horowitz (1996), which allows us to obtain twostep GMM refinements by resampling non-overlapping blocks of observations in order to accommodate potential data dependence. The bias-corrected bootstrap estimates were quite similar to those obtained using the CUE and GEL estimation procedures.
} 
weak - instruments ${ }^{6}$. Also, though GEL estimation is more efficient, if weak identification is pervasive, the t-ratios from Table 1 may not be valid. Thus, to circumvent this problem, we employ the identification-robust statistics of Kleibergen (2005), which are valid regardless of whether the parameters are strongly or weakly identified, as well as being robust under many weak moment conditions.

For conciseness, we consider in more detail the forward-looking specification studied in RW. We can focus on the main parameter of interest and conduct tests for $H_{0}: \alpha_{c}=\bar{\alpha}_{c}$ without assuming identification, by considering subset tests with Kleibergen-type statistics, as discussed in Kleibergen and Mavroeidis (2009a and b). These authors show that testing can be carried out by estimating with the CUE the subset of unrestricted parameters $\boldsymbol{\theta}=\{\beta, \theta\}$ for given values of $\alpha_{c}, \hat{\boldsymbol{\theta}}\left(\alpha_{c}\right)$. We thus obtain confidence sets for $\alpha_{c}$ by performing a grid search over its parameter space $^{7}$, testing $H_{0}: \alpha_{c}=\bar{\alpha}_{c}$ and collecting the values $\bar{\alpha}_{c}$ for which the p-value exceeds a joint $5 \%$ significance level. We follow the suggestion of Kleibergen (2005) by combining his $K$ statistic (for which a $\chi^{2}(1)$ limiting distribution is used, see Kleibergen and Mavroeidis, 2009a, Theorem 1 , for example) with the asymptotically independent $J$ statistic for overidentifying restrictions, distributed as $\chi^{2}(m-p)$. For the combined $J$ - $K$ test, denoted $K^{*}$, we use a significance level of $1 \%$ for the $J$-test and $4 \%$ for the $K$-test, therefore emphasizing simple parameter hypothesis testing, see Kleibergen (2005) for details. For the EL estimator, we use the LM version of the Kleibergen-type test proposed by Guggenberger and Smith (2008), which was found to have advantageous finite-sample properties in their Monte Carlo study.

We illustrate this identification-robust approach by plotting in Figure 1 the p-values sequence of the $K^{*}$ statistic for the grid of values $\bar{\alpha}_{c}$ when $\{\beta, \theta\}$ is fixed at the benchmark case of $\{0.99,0.75\}$. We can observe that the region for which the null $H_{0}: \alpha_{c}=\bar{\alpha}_{c}$ is not rejected is formed by, approximately, the interval $(-0.1,0.4)$. We also obtained confidence sets for $\alpha_{c}$ considering all possible values of $\theta$ by concentrating this parameter out. We report in the top panel of Table 2 the intervals for both parameters for which the $K^{*}$ statistic does not reject the joint null, for both the CUE and EL estimators. We can see that, while the values for $\theta$ tend to

\footnotetext{
${ }^{6}$ While using fewer instruments may alleviate the bias of the 2-step GMM estimator, it does not remedy weak identification if the remaining instruments are weak, see Han and Phillips (2006) and Newey and Windmeijer (2009) for a discussion on GMM estimation with many (weak) moment conditions. In our analysis, we follow the recommendation of Kleibergen and Mavroeidis (2009a, p. 307) and present results based on instrument set B, as there may be size distortions when many instruments are used together with a HAC estimator.

${ }^{7}$ We choose the interval $(-0.5,1.5)$, with increments of 0.01 , thus including values close to 0 (no cost channel) and values larger than 1 , consistent with estimates presented in RW and CHS.
} 
be above 0.7 , the identification-robust confidence interval is completely uninformative for $\alpha_{c}$, as it contains the entire parameter space considered in the grid. Indeed, it contains economically relevant values for $\alpha_{c}$, but it also includes the case of no cost channel. Thus, even when potential weak parameter identification is taken into account, the statistical evidence does not rule out the absence of a cost channel effect.

Nevertheless, we conducted further robustness checks, also presented in Table 2. First, we note that the differences reported in our results cannot be attributed to the use of a different sample period. When we restrict the sample size to be the same as in RW (1960:1 to 2001:1), the subset-based identification-robust confidence intervals are not qualitatively different from those obtained above, as $\alpha_{c}$ is always insignificant. We also consider estimation of the cost channel with the sample starting in 1981, thus excluding the two oil shocks as in CHS, but again the same pattern emerges (in the case of the EL statistic, the overidentifying restrictions are rejected, hence the empty confidence set). Secondly, we re-estimated $\alpha_{c}$ using different variancecovariance matrices for the CUE. As argued before, the choice of the weighting matrix for GMM estimation may affect inference on the NKPC, in particular whether or not the cost channel is statistically significant. Clearly, the results in the bottom panel of Table 2 indicate that the use of different kernels to obtain the optimal weighting matrix does not alter our previous analysis. Therefore, we can conclude that previous results in the literature seem to be method-specific and do not withstand a more thorough sensitivity analysis.

\section{Conclusion}

In this paper we re-examine the empirical relevance of the cost channel of monetary transmission. We employ newly developed efficient inference techniques that are not sensitive to the specification of the orthogonality conditions and are robust to weak parameter identification. We conclude that, in a single-equation framework, the cost channel effect is poorly identified, suggesting that, while the data is consistent with the presence of a cost channel, one cannot rule out zero interest rate effects. This helps explaining the conflicting results reported in the literature. Indeed, our evidence does not fully corroborate the results previously reported in Ravenna and Walsh (2006) and Chowdhury et al. (2006). It appears that once we adopt more appropriate method-of-moments procedures, the conclusions are also consistent with the findings of the VAR-based study of Mojon (2008), as well as of Rabanal (2007), obtained with Bayesian methods in the context of a full DSGE model. 
However, given the identification problems we highlight in this paper, we do not claim that a cost channel effect is inexistent, as one cannot exclude the possibility of a substantial cost channel either. Indeed, what we show is that, for the particular periods considered, its aggregate effect is not sufficiently noticeable. This, in turn, suggests a few directions for further investigation. On one hand, it may well be the case the cost channel effect becomes more prominent at particular stages of the business cycle, as suggested by the results in Tillmann (2008), but is then averaged out when longer periods are considered. Also, propagation mechanisms of monetary shocks are likely to have disparate effects across different industries, as shown by Barth and Ramey (2001) and Dale and Haldane (1995). Finally, it is also conceivable that these channels become more relevant in developing economies, with a less stable history of inflation and less efficient financial markets. Further research is therefore required.

\section{References}

[1] Anatolyev, S. (2005), GMM, GEL, Serial Correlation and Asymptotic Bias, Econometrica, 73, 983-1002.

[2] Andrews, D. W. K. (1991), Heteroskedasticity and Autocorrelation Consistent Covariance Matrix Estimator, Econometrica, 59, 817-858.

[3] Barth, M. J. and Ramey, V. A. (2001), The Cost Channel of Monetary Transmission, NBER Macroeconomics Annual, 16, 199-240.

[4] Chowdhury, I., Hoffmann, M. and Schabert, A. (2006), Inflation dynamics and the cost channel of monetary transmission, European Economic Review, 50, 995-1016.

[5] Christiano, L. J., Eichenbaum, M. and Evans, C. L. (2005), Nominal Rigidities and the Dynamic Effects of a Shock to Monetary Policy, Journal of Political Economy, 113, 1-45.

[6] Dale, S. and Haldane, A. G. (1995), Interest rates and the channels of monetary transmission: Some sectoral estimates, European Economic Review, 39, 1611-1626.

[7] Gali, J. and Gertler, M. (1999), Inflation dynamics: A structural econometric analysis, Journal of Monetary Economics, 44, 195-222.

[8] Guggenberger, P. and Smith, R. J. (2008), Generalized Empirical Likelihood Tests in Time Series Models With Potential Identification Failure, Journal of Econometrics, 142, 134-161. 
[9] Hall, P. and Horowitz, J. L. (1996), Bootstrap Critical Values for Tests Based on Generalized-Method-of-Moments Estimators, Econometrica, 64, 891-916.

[10] Han, C. and Phillips, P. C. B. (2006), GMM with Many Moment Conditions, Econometrica, $74,147-192$.

[11] Hansen, L. P., Heaton, J. and Yaron, A. (1996), Finite-Sample Properties of Some Alternative GMM estimators, Journal of Business and Economic Statistics, 1996, 14, 262-80.

[12] Kitamura, Y. and Stutzer, M. (1997), An Information-Theoretic Alternative to Generalized Method of Moments Estimation, Econometrica, 65, 861-874.

[13] Kleibergen, F. (2005), Testing parameters in GMM without assuming that they are identified, Econometrica, 73, 1103-1123.

[14] Kleibergen, F. and Mavroeidis, S. (2009a), Weak Instrument Robust Tests in GMM and the New Keynesian Phillips Curve, Journal of Business Economics and Statistics, 27, 293-311.

[15] Kleibergen, F. and Mavroeidis, S. (2009b), Inferences on subset of parameters in GMM without assuming identification, manuscript.

[16] Mavroeidis, S. (2005), Identification Issues in Forward-Looking Models Estimated by GMM, with an Application to the Phillips Curve, Journal of Money, Credit and Banking, 37, 421448.

[17] Mojon, B. (2008), When did unsystematic monetary policy have an effect on inflation?, European Economic Review, 52, 487-497.

[18] Newey, W. K. and Smith, R. J. (2004), Higher Order Properties of GMM and Generalized Empirical Likelihood Estimators, Econometrica, 72, 219-255.

[19] Newey, W. K. and Windmeijer, F. (2009), Generalized Method of Moments with Many Weak Moment Conditions, Econometrica, 77, 687-719.

[20] Rabanal, P. (2007), Does inflation increase after a monetary policy tightening? Answers based on an estimated DSGE model, Journal of Economic Dynamics and Control, 31, 906-937.

[21] Ravenna, F. and Walsh, C. E. (2006), Optimal monetary policy with the cost channel, Journal of Monetary Economics, 53, 199-216. 
[22] Stock, J. H. and Wright, J. H. (2000), GMM With Weak Identification, Econometrica, 68, 1055-1096.

[23] Tillmann, P. (2008), Does the Cost Channel of Monetary Transmission Explain Inflation Dynamics, Journal of Economic Dynamics and Control, 32, 2723-2744.

[24] Tillmann, P. (2009), Optimal Monetary Policy with an Uncertain Cost Channel, Journal of Money, Credit and Banking, 41, 885-906.

\section{Appendix}


Table 1: GEL estimates of the interest-rate-augmented NKPC

\begin{tabular}{|c|c|c|c|c|c|c|}
\hline \multicolumn{2}{|c|}{ Instrument set A } & $\beta$ & $\theta$ & $\alpha_{c}$ & $\gamma$ & $\underset{(\mathrm{p} \text {-values })}{J \text {-test }}$ \\
\hline \multicolumn{7}{|c|}{ Forward-looking (eq. 1) } \\
\hline \multirow[t]{2}{*}{ Restricted } & CUE & $\begin{array}{l}1.009 \\
(0.025)\end{array}$ & $\begin{array}{l}0.891 \\
(0.184)\end{array}$ & - & - & 0.318 \\
\hline & EL & $\begin{array}{l}0.972 \\
(0.012)\end{array}$ & $\begin{array}{l}0.878 \\
(0.079)\end{array}$ & - & - & 0.392 \\
\hline \multirow[t]{2}{*}{ Unrestricted } & CUE & $\begin{array}{l}1.004 \\
(0.025)\end{array}$ & $\begin{array}{l}0.836 \\
(0.106)\end{array}$ & $\begin{array}{l}0.326 \# \\
(0.516)\end{array}$ & - & 0.361 \\
\hline & EL & $\begin{array}{l}0.967 \\
(0.012)\end{array}$ & $\begin{array}{l}0.892 \\
(0.092)\end{array}$ & $\begin{array}{l}0.393 \\
(0.663)\end{array}$ & - & 0.369 \\
\hline \multicolumn{7}{|c|}{ Hybrid (eq. 2) } \\
\hline \multirow[t]{2}{*}{ Restricted } & CUE & $\begin{array}{l}0.965 \\
(0.044)\end{array}$ & $\begin{array}{l}0.876 \\
(0.268)\end{array}$ & - & $\begin{array}{l}0.397 \\
(0.165)\end{array}$ & 0.762 \\
\hline & EL & $\begin{array}{l}0.967 \\
(0.015)\end{array}$ & $\begin{array}{c}0.834 \\
(0.08)\end{array}$ & - & $\begin{array}{l}0.317 \\
(0.057)\end{array}$ & 0.833 \\
\hline \multirow[t]{2}{*}{ Unrestricted } & CUE & $\begin{array}{l}0.966 \\
(0.045)\end{array}$ & $\begin{array}{l}0.861 \\
(0.235)\end{array}$ & $\begin{array}{l}0.190 \# \\
(0.838)\end{array}$ & $\begin{array}{l}0.392 \\
(0.157)\end{array}$ & 0.723 \\
\hline & EL & $\begin{array}{l}0.966 \\
(0.016)\end{array}$ & $\begin{array}{l}0.841 \\
(0.082)\end{array}$ & $\begin{array}{l}0.131 \# \\
(0.194)\end{array}$ & $\begin{array}{l}0.309 \\
(0.058)\end{array}$ & 0.800 \\
\hline
\end{tabular}

Instrument set B

Forward-looking (eq. 1)

\begin{tabular}{|c|c|c|c|c|c|c|}
\hline \multirow[t]{2}{*}{ Restricted } & CUE & $\begin{array}{l}0.995 \\
(0.026)\end{array}$ & $\begin{array}{l}0.950 \\
(0.469)\end{array}$ & - & - & 0.189 \\
\hline & EL & $\begin{array}{l}0.974 \\
(0.010)\end{array}$ & $\begin{array}{l}0.950 \\
(0.192)\end{array}$ & - & - & 0.106 \\
\hline \multirow[t]{2}{*}{ Unrestricted } & CUE & $\begin{array}{l}0.987 \\
(0.026)\end{array}$ & $\begin{array}{l}0.846 \\
(0.125)\end{array}$ & $\begin{array}{l}0.371 \# \\
(0.685)\end{array}$ & - & 0.220 \\
\hline & EL & $\begin{array}{l}0.964 \\
(0.010)\end{array}$ & 0.943 & $1.372^{\#}$ & - & 0.136 \\
\hline
\end{tabular}

Hybrid (eq. 2)

\begin{tabular}{|c|c|c|c|c|c|c|}
\hline \multirow[t]{2}{*}{ Restricted } & CUE & $\begin{array}{l}0.957 \\
(0.052)\end{array}$ & $\begin{array}{l}0.816 \\
(0.190)\end{array}$ & - & $\begin{array}{l}0.429 \\
(0.152)\end{array}$ & 0.627 \\
\hline & EL & $\begin{array}{l}0.973 \\
(0.013)\end{array}$ & $\begin{array}{l}0.773 \\
(0.054)\end{array}$ & - & $\begin{array}{l}0.317 \\
(0.055)\end{array}$ & 0.305 \\
\hline \multirow[t]{2}{*}{ Unrestricted } & CUE & $\begin{array}{c}0.961 \\
(0.052)\end{array}$ & $\begin{array}{l}0.815 \\
(0.185)\end{array}$ & $\begin{array}{l}0.087 \text { \# } \\
(0.379)\end{array}$ & $\begin{array}{l}0.415 \\
(0.152)\end{array}$ & 0.561 \\
\hline & EL & $\begin{array}{l}0.966 \\
(0.012)\end{array}$ & $\begin{array}{l}0.833 \\
(0.067)\end{array}$ & $\begin{array}{l}0.275 \# \\
(0.247)\end{array}$ & $\begin{array}{l}0.313 \\
(0.062)\end{array}$ & 0.326 \\
\hline
\end{tabular}

Note - standard errors in brackets; ${ }^{\#}$ means statistically insignificant 
Table 2: 95\% identification-robust confidence intervals

\begin{tabular}{|c|c|c|c|}
\hline Bivariate & $\theta$ & $\alpha_{c}$ & \\
\hline CUE & {$[0.72,0.99]$} & {$[-0.5,1.5]$} & \\
\hline EL & {$[0.67,0.99]$} & {$[-0.5,1.5]$} & \\
\hline Samples: & 1960:1-2004:1 & 1960:1-2001:1 & 1981:1-2004:4 \\
\hline CUE & $\begin{array}{c}0.371 \\
{[-0.5 ; 1.5]}\end{array}$ & $\begin{array}{c}0.441 \\
{[-0.5 ; 1.5]}\end{array}$ & $\begin{array}{c}0.390 \\
{[-0.5 ; 0.75]}\end{array}$ \\
\hline \multirow[t]{2}{*}{ EL } & $\begin{array}{c}1.372 \\
{[-0.3 ; 1.5]}\end{array}$ & $\begin{array}{c}0.758 \\
{[-0.46 ; 1.5]}\end{array}$ & $\varnothing$ \\
\hline & Parzen & Quadratic Spectral & Bartlett \\
\hline HAC estimator & $\begin{array}{c}0.593 \\
{[-0.36 ; 0.21]}\end{array}$ & $\begin{array}{c}0.396 \\
{[-0.5 ; 1.5]}\end{array}$ & $\begin{array}{c}0.185 \\
{[-0.5 ; 1.5]}\end{array}$ \\
\hline
\end{tabular}

Notes: $\varnothing$ - overidentifying restrictions always rejected 